\title{
Rechnen in der Wolke
}

Rechnen in der Wolke, d. h. zu jeder Zeit und von fast jedem Ort auf seine Daten zugreifen und sich sozusagen in einer virtuellen Datenwolke bewegen zu können, das ist die Zukunftsvision des "Cloud Computing". Ursprünglich handelt es sich dabei um einen Begriff aus der Informationstechnik. Gemeint ist damit die Möglichkeit, Speicherkapazitäten, Rechenleistung und Software über das Internet zu erhalten, für die der Nutzer je nach Umfang, Nutzungsdauer und Nutzungsart zahlt. Der Vorteil: Auf dem eigenen Rechner können Rechenarbeit und Speicherplatz eingespart und dadurch selbst mit leistungsschwachen Rechnern die neuesten Verarbeitungsprogramme genutzt werden. Nutzer werden außerdem nicht mehr mit der langwierigen Installation und regelmäßigen Pflege von Software belastet. Hohe Investitionskosten für neue Programme sollen den Unternehmen erspart bleiben, und es soll ihnen überdies eine schnellere Anpassung der Informationstechnik (Zahl der Lizenzen, Art der Programme) an Änderungen der Geschäftslage oder -ausrichtung ermöglicht werden. Problem des "Cloud Computing" ist dabei, dass die Daten - teilweise in Einzelteile zerlegt - für den Nutzer nicht nachvollziehbar auf über die ganze Welt verteilten Servern gespeichert werden. Bereits heute wird der Umgang mit dem Internet durch "Cloud Computing" beeinflusst, so wendet z. B. Google Maps "Cloud Computing" bereits unternehmensintern an.' Unterschieden werden kann in diesem Zusammenhang zwischen sogenannten „Private Clouds" für geschlossene Nutzergruppen und "Public Clouds", die für eine große Anzahl verschiedener Nutzer zurVerfügung stehen. Daneben finden sich häufig Nutzungskombinationen von Private Clouds, Public Clouds und einer traditionellen IT-Umgebung („Hybrid Clouds").

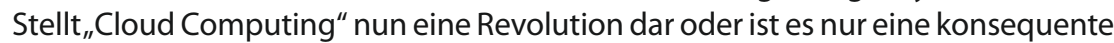
Fortsetzung des bereits weit verbreiteten "verteilten Rechnens" und der Tendenz zum Outsourcing? Ist "Cloud Computing" die Zukunft des Internets oder handelt es sich doch nur um einen vergänglichen IT-Modebegriff? Dies sind zurzeit die am häufigsten diskutierten Fragen in den allgemeinen Berichten und Aufsätzen zum "Cloud Computing". Dabei liegen die zentralen Probleme im rechtlichen Bereich, in der Sicherstellung des Datenschutzes und der Datensicherheit, wie der Beitrag von Thilo Weichert im Überblick zeigt. Zu berücksichtigen ist außerdem, dass es sich beim "Cloud Computing" im Kern um eine Outsourcing-Technik handelt. Dadurch ergeben sich vor allem Fragestellungen zur Auftragsdatenverarbeitung sowie zur Funktionsübertragung. Die Auswirkungen der letzten Änderungen im BDSG zur Auftragsdatenverarbeitung sind Gegenstand der Ausführungen von Thomas Hoeren. Daneben stellt gerade die Etablierung eines geeigneten technischen und organisatorischen Identitäts- und Zugriffsmanagements eine wesentliche Voraussetzung dafür dar, dass Unternehmen die Einhaltung von Datenschutzbestimmungen und Datensicherheit beim Einsatz von "Cloud Computing“ gewährleisten können. Philip Laue und Oliver Stiemerling zeigen die damit verbundenen technisch-organisatorischen Probleme sowie Risiken auf und diskutieren hierzu Lösungsansätze.

\section{Britta Alexandra Mester}

